\title{
Comparative Evaluation of a New Sensor for Superparamagnetic Iron Oxide Nanoparticles in a Molecular Communication Setting
}

This paper was downloaded from TechRxiv (https://www.techrxiv.org).

\section{LICENSE}

CC BY-NC-SA 4.0

SUBMISSION DATE / POSTED DATE

$11-01-2021 / 13-01-2021$

\section{CITATION}

Bartunik, Max; Unterweger, Harald; Alexiou, Christoph; Schober, Robert; Lübke, Maximilian; Fischer, Georg; et al. (2021): Comparative Evaluation of a New Sensor for Superparamagnetic Iron Oxide Nanoparticles in a Molecular Communication Setting. TechRxiv. Preprint. https://doi.org/10.36227/techrxiv.13554401.v1

$\mathrm{DOI}$ 


\title{
Comparative Evaluation of a New Sensor for Superparamagnetic Iron Oxide Nanoparticles in a Molecular Communication Setting
}

\author{
Max Bartunik ${ }^{1[0000-0002-3033-5798]}$, Harald Unterweger ${ }^{20000-0002-8335-0692]}$, \\ Christoph Alexiou ${ }^{2[0000-0003-2220-6790]}$, Robert Schober ${ }^{3}$, Maximilian Lübke ${ }^{1}$, \\ Georg Fischer ${ }^{1[0000-0002-4033-2005]}$, and Jens Kirchner1 [0000-0002-8623-9551] \\ 1 Institute for Electronis Engineering, Friedrich-Alexander-Universität \\ Erlangen-Nürnberg (FAU), Germany \\ max.bartunik@fau.de \\ jens.kirchner@fau.de \\ 2 Section of Experimental Oncology and Nanomedicine (SEON), University Hospital \\ Erlangen, Germany \\ ${ }^{3}$ Institute for Digital Communications, Friedrich-Alexander-Universität \\ Erlangen-Nürnberg, Germany
}

\begin{abstract}
Testbeds are required to assess concepts and devices in the context of molecular communication. These allow the observation of real-life phenomena in a controlled environment and therefore present the basis of future work. A testbed using superparamagnetic iron oxide nanoparticles (SPIONs) as information carriers was constructed with regard to this context and requires a sensitive receiver for the detection of SPIONs.

This paper focusses on the comparison between a newly presented device (inductance sensor), a previously constructed SPION sensor (resonance bridge), and a commercial susceptometer as reference. The new inductance sensor is intended to improve on a low sensitivity achieved with the previous device and restrictions with respect to sample rate and measurement aperture encountered with the susceptometer. The signal-to-noise ratio (SNR) for each device is assessed at a variety of SPION concentrations. Furthermore, the sensors bit error rates (BER) for a random bit sequence are determined.

The results show the device based on an inductance sensor to be the most promising for further investigation as values both for BER and SNR exceed those of the resonance bridge while providing a sufficiently high sample rate. On average the SNR of the new device is $13 \mathrm{~dB}$ higher while the BER for the worst transmission scenario is $9 \%$ lower. The commercial susceptometer, although returning the highest SNR, lacks adaptability for the given use case.
\end{abstract}

Keywords: Molecular Communication · Superparamagnetic Iron Oxide Nanoparticles · SPION · Testbed · Susceptometer · Resonance Bridge · Inductance Sensor $\cdot \mathrm{SNR} \cdot \mathrm{BER}$ 


\section{Introduction}

Molecular communication, with the aim of transferring information using molecules or particles in the nanoscale, poses various new challenges like the development of adequate coding schemes or the assessment of new physical effects in the context of data transmission. An overview of different strategies for these challenges in molecular communication can be found in [5,9]. One essential aspect of exploring both phenomena of a physical nature and evaluating channel coding methods is the use of testbeds that facilitate the observation of relevant effects under controlled and reproducible conditions in a representative model.

First testbeds proposed for molecular communication used alcohol-based transmission $[2,6,11]$ or acids/bases as information carriers $[3,4,7]$. The authors in [10] present an alternative testbed using superparamagnetic iron oxide nanoparticles (SPIONs) as transmission particles. These were originally developed for applications in cancer therapy and as such are biocompatible, enabling new use cases in medical applications. The detection of particles can be achieved by measuring the change of a magnetic field generated by a coil wound around the transmission channel [1].

Finding an adequate detector for SPIONs in the given testbed setup is a vital step to achieving high-quality transmission under noisy conditions. Besides a sufficient sample rate, high sensitivity is required to accurately determine low concentrations of SPIONs.

While using a commercial susceptometer (MS2/MS3 from Bartington Instruments Ltd.) for the detection of SPIONs, the authors of [10] discovered various downsides of the device. Having a fixed measurement aperture on the one hand results in a potential loss of sensitivity when the channel is smaller than the measurement coil, and on the other hand does not allow for use of a channel wider than the given measurement opening. Furthermore, as the device was not designed for continuous measurements it has a maximal sample rate of $10 \mathrm{Sa} \mathrm{s}^{-1}$, insufficient for the use case [1]. For these reasons the authors of [1] proposed a new device that allows for use of custom made coils and sampling with a higher rate. The device of own making however showed less sensitivity at low particle concentrations and has the significant disadvantage of a complicated tuning procedure that causes inconsistent measurement accuracy.

Here, we present a third detector device based on an inductance sensor (LDC1612 from Texas Instruments) that also allows for the use of custom coils and does not require manual tuning to a resonance frequency. Additionally, the device facilitates differential measurement and reduction of systematic environment noise, such as temperature drift, by use of two detection channels similar to the device in [1], unlike the commercial susceptometer.

We will outline the used testbed and present the different sensor devices in the following sections. Then, an in-depth comparison of all three receivers, using the existing testbed, in respect to sensitivity and achievable data transmission accuracy is conducted. We conclude the article with an outlook on future sensor and testbed developments. 


\section{Setup}

To evaluate the different sensors for the given use case we used a testbed utilising SPIONs as information carriers in a channel with constant background flow. This was developed in previous work and has been published in [1] and [10].

\section{$2.1 \quad$ SPIONs}

The SPIONs used as information carriers were synthesized by the Section for Experimental Oncology and Nanomedicine (SEON) of the University Hospital Erlangen. They were originally developed for biomedical applications and have a coating of lauric acid, which can be employed for use in drug targeting. With a hydrodynamic diameter of $50 \mathrm{~nm}$ they have a specific susceptibility of $8.78 \times 10^{-3}$ for $1 \mathrm{mg} \mathrm{Fe} \mathrm{ml}{ }^{-1}$. They are dispersed in aqueous solution with a particle concentration of $9 \times 10^{13}$ particles $\mathrm{ml}^{-1}$ at $10 \mathrm{mg} \mathrm{Fe} \mathrm{ml}^{-1}$.

\section{$2.2 \quad$ Testbed}

Transmission in the testbed is achieved by injecting SPIONs into a tube with a constant background flow of water. The information carriers are sourced from a syringe containing a specific stock concentration of SPIONs in aqueous suspension. A peristaltic computer controlled pump (Ismatec ${ }^{\circledR}$ ISM596D) is used to inject a volume of this solution into the background channel at a y-connector that is placed so that the injection occurs against the direction of flow, as can be seen in Figure 1, to reduce washing-out of particles over time. The transmission channel itself consists of a tube with an inner diameter of $1.52 \mathrm{~mm}$ and is driven by a second peristaltic pump (Ismatec ${ }^{\circledR}$ ISM831C). The distance between the injection point and the appropriate coil was chosen to be $5 \mathrm{~cm}$.

Generally, the setup can be modelled as a channel with laminar flow, describing different flow speeds throughout the channel width with a radial dependency. Therefore SPIONs in the centre of the tube move fastest, while particles at the edge are transported very slowly. This causes an axial distribution of the injected SPIONs with an initial bulk quantity at the front followed by a trail with declining concentration.

\section{Detector Devices}

A total of three different devices for the detection of SPIONs are assessed in this paper. All detectors in turn rely on the magnetic properties of the SPIONs, which being superparamagnetic have a high susceptibility. As the nanoparticles are received at the detector they pass through a coil and act as a magnetic core. A change of inductance value $\Delta L$ for the detector coil, as can be described by

$$
\Delta L=\chi \frac{\mu_{0} A N^{2}}{l}
$$




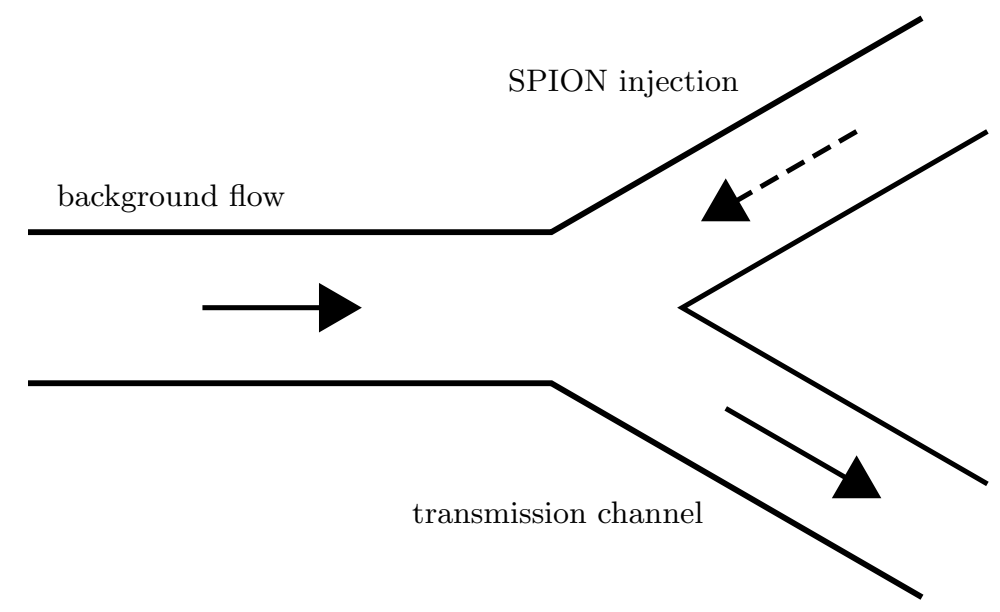

Fig. 1. Schematic model of the y-connector used for injection of SPIONs into the background flow. The injection tube faces towards the flow. All connected tubes have an equal width.

for the given susceptibility $\chi$ of the core $[8$, pp. 261,315], is the result. Here $A$ is the cross-section of the coil, $N$ is the amount of windings and $l$ is the length of the coil. Clearly, as the susceptibility $\chi$ of the core material changes (i.e. SPIONs pass through the coil) the inductance of the coil changes proportionally. This change can also be described as shift of resonant frequency in a parallel resonator circuit with a capacitor $C$ :

$$
f_{\mathrm{Res}}=\frac{1}{2 \pi \sqrt{L C}}
$$

As the particles are distributed by laminar flow in the transmission channel and do not pass through the detector as homogenous core, the impulse response shows an appropriate short rising edge followed by a slow decay [10].

\subsection{Commercial Susceptometer}

We applied the commercial susceptometer system MS2/MS3 from Bartington Instruments Ltd. as a reference device. The sensor consists of the actual susceptometer MS3 and a selection of attachable sensors (MS2). In our case we used the sensor MS2G, which has a cylindrical measurement aperture with a radius of $4.25 \mathrm{~mm}$ and a height of $28 \mathrm{~mm}$. The detector coil, and therefore the area of measurement, spans $5 \mathrm{~mm}$ and is operated at a frequency of $1.3 \mathrm{kHz}$.

\subsection{Resonance Bridge}

The first device we inspected for comparison uses a resonance bridge for differential measurement between two detector coils. Typically, one coil is used as 
reference to reduce systematic noise caused by temperature changes or other environmental influences. This device was developed at the Institute for Electronics Engineering of the FAU and was published in [1]. It consists of a fully independent printed circuit board $(\mathrm{PCB})$ that only requires a regular $5 \mathrm{~V}$ power supply, as can be drawn through the USB standard. Detector coils, operated at $10 \mathrm{MHz}$, are attached externally via SMA-connectors and as such can easily be interchanged to fit the channel requirements. An analog DC-signal representing the measured susceptibility is obtained as output and can be digitally acquired through an analog-digital-converter (ADC). The PCB has a size of $7 \mathrm{~cm}$ by $7 \mathrm{~cm}$.

The required detector coils were custom made using bondable enamelled wire to allow adaptation to the transmission channel girth and ensure mechanical stability. They each consist of 20 windings in one layer with a length of approximately $20 \mathrm{~mm}$. Figure 2 shows the coils, mounted on a PCB and equipped with SMA-connectors for ease of use.

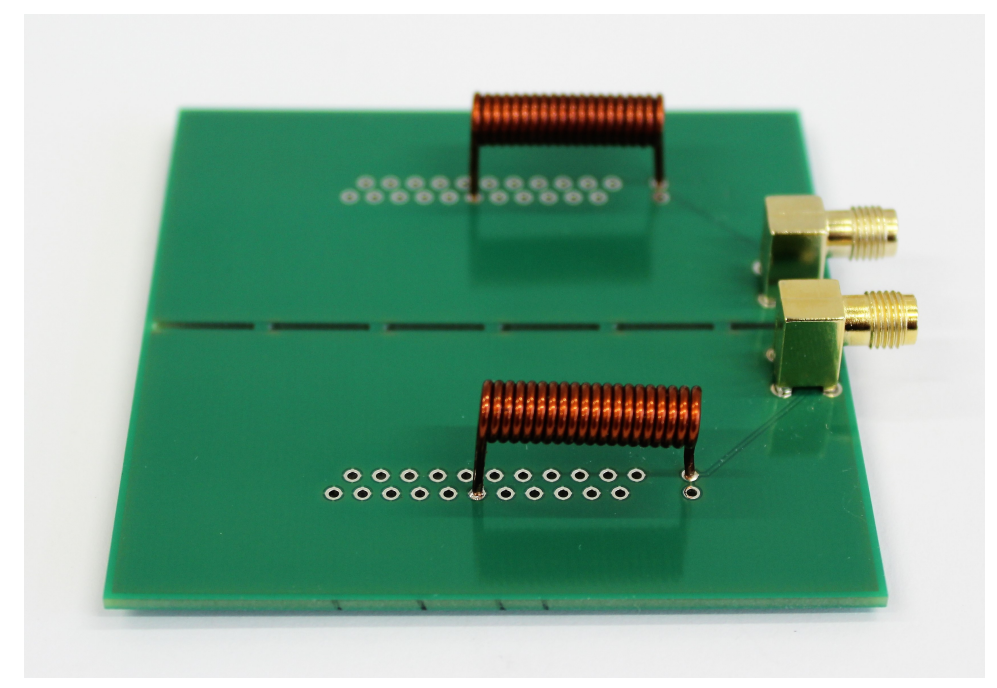

Fig. 2. PCB with detector coils used for measurements, as published in [1].

Before measurements, the devices channels have to be manually tuned via adjustable capacitors to the resonance frequency of $10 \mathrm{MHz}$ and to each other. The measurement precision depends greatly on the accuracy of this tuning procedure, which requires an oscilloscope.

\subsection{Inductance Sensor}

Finally, the inductance sensor LDC1612 from Texas Instruments was also applied in the testbed. In contrast to the resonance bridge it does not inherently perform a differential measurement but rather determines the resonant frequency 
of a connected circuit digitally. The sensor provides two measurement channels, which can be variably driven within the range of $1 \mathrm{kHz}$ to $10 \mathrm{MHz}$. It was operated in a setup using the evaluation module LDC1612 EVM provided by Texas Instruments and was fitted with SMA-connectors to facilitate use with custom made coils, as described in section 3.2. Figure 3 shows the device mounted in a custom made housing for mechanical protection.

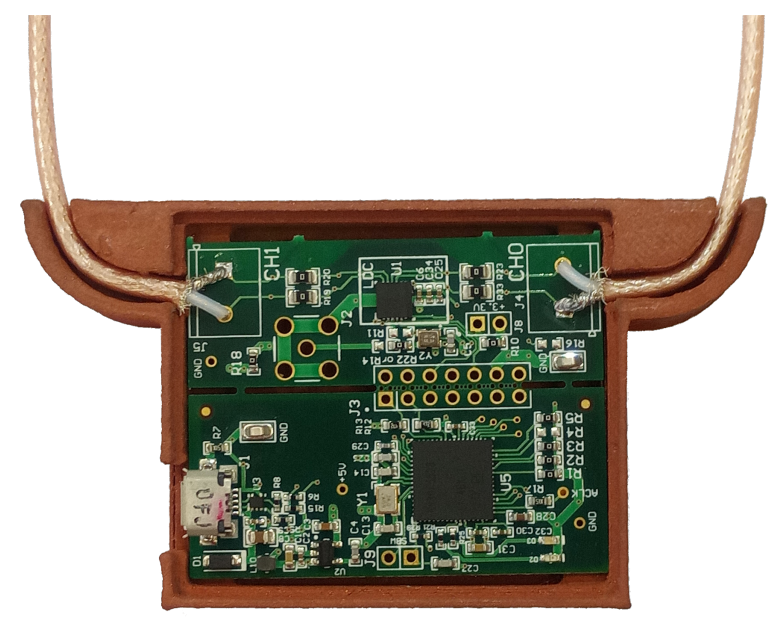

Fig. 3. Inductance Sensor consisting of the evaluation module LDC1612 EVM with two SMA-connectors mounted in a housing.

In first function tests a systematic drift of the measurement values could be observed on both channels. To counteract this shifting offset, the evaluation module was fitted with temperature stable capacitors (rating C0G (NP0)) in the measurement circuit and a differential detection between the two symmetrically drifting channels was implemented in software.

\section{Evaluation Procedure}

\subsection{Bit detection}

The capabilities of each receiver were initially assessed by transmitting 80 bits of random data (3).

$\begin{array}{lllll}11111001 & 11110010 & 00111000 & 10010011 & 01110010 \\ 00010000 & 11111011 & 01000111 & 10110101 & 01100110\end{array}$


Each '1' bit was coded as an injection of SPION-suspension into the background flow, whereas no SPIONs were injected during a ' 0 ' symbol period. Using the software Matlab (MathWorks) an automatic detection of the received signal was implemented. First, a moving averaging filter was applied to the results of the resonance bridge and inductance sensor resulting in an effective sample rate of $10 \mathrm{Sa} \mathrm{s}^{-1}$, similar to that of the susceptometer. Then for each measurement the average between maximal and minimal signal value was set as a threshold for bit detection. Next the symbol intervals with a known duration of $1 \mathrm{~s}$ were derived from the first rising edge. To allow for inconsistencies in the sampling rate of the susceptometer the symbol intervals were resynchronised at every rising edge. Finally, the corresponding bit was set to ' 1 ' if the threshold was met for at least $30 \%$ of the symbol interval.

Measurements were performed using different injection volumes (i.e., 7.01, 14.0, 28.0, 56.1, 84.1 $\mathrm{\mu l}$ ) to transmit the random sequence. Bit error rates (BERs) were calculated using the algorithm described above to demonstrate the capabilities of each individual sensor.

\subsection{Sensitivity}

A relevant factor for sensor quality in the molecular communication setup is sensitivity as confident detection of low concentrations of SPIONs is desired. The signal-to-noise ratio (SNR) of the individual devices was used as an index for sensitivity. To this end a sequence of three individual injections was performed and the maximal amplitude of the measured signal $\hat{U}_{\mathrm{S}}$ recorded and averaged over the three injections. In turn the noise $\hat{U}_{\mathrm{N}}$ was determined during transmission of a ' 0 ' bit (no injection of SPIONs). The SNR value is the result of the relation

$$
\mathrm{SNR}_{\mathrm{dB}}=20 \log \frac{\hat{U}_{\mathrm{S}}}{\hat{U}_{\mathrm{N}}}
$$

As the injected SPIONs are distributed due to laminar flow, and reduced volume results in a smaller portion of the detector coil being filled with SPIONs at once, less injection volume corresponds to a lower signal amplitude.

\subsection{Bit detection at varying symbol intervals}

To assess the influence of sensitivity and achievable sample rate for the individual devices, the random sequence as given by (3) was transmitted using varying symbol intervals ranging from $0.2 \mathrm{~s}$ to $1 \mathrm{~s}$. A symbol interval of less than $0.2 \mathrm{~s}$ is not possible due to the maximal speed of the injection pump.

The principal algorithm described in 4.1 was again used to calculate a BER. However, in this case data points were added by means of interpolation to allow for detection of the shorter symbol intervals. Without interpolation an interval of $0.2 \mathrm{~s}$ in the case of the susceptometer, with an effective sample rate of less than $10 \mathrm{Sa} \mathrm{s}^{-1}$, would be represented by less than two data points. The samples were therefore increased to $100 \mathrm{Sa} \mathrm{s}^{-1}$. Furthermore, the threshold was set to 
$80 \%$ of the average between maximal and minimal amplitude to allow for lower signal levels due to the use of smaller injection volumes $(28.0 \mathrm{\mu l}$ and $14.0 \mathrm{\mu l})$.

\section{$5 \quad$ Results}

\subsection{Bit detection}

Figure 4 shows the received signal after averaging for the three detection devices at an injection volume of $84.1 \mathrm{\mu l}$. The measured signals show the expected behaviour of a steep rising edge and a slow signal decay due to laminar flow.

Table 1 shows the calculated BER for each sensor at various injection volumes. For a high injection volume a BER of $0 \%$ and therefore perfect transmission was achieved for all devices. The expected result of rising bit errors when the injection volume and therefore the signal amplitude is reduced can also be observed with all three devices. At the lowest injection volume of $7.01 \mu \mathrm{l} \mathrm{bit}$ errors are very dominant with error rates close to random data $(50 \%)$.

The inductance sensor consistently shows a better BER than the resonance bridge, which already has a significantly high BER at an injection volume of $28.0 \mathrm{pl}$. Although the inductance sensor has a higher BER than the susceptometer for injections of $28.0 \mathrm{\mu l}$ and $14.0 \mathrm{\mu l}$, more bits were detected correctly for the lowest injection volume $(7.01 \mathrm{\mu l})$. An explanation for this can be found in the very low sampling rate of the susceptometer causing insufficient representation of the short pulse at the detector.

Table 1. BERs for various injection volumes derived from the transmission of 80 random bits. The worst possible BER is $50 \%$, equivalent to random data.

\begin{tabular}{cccc}
\hline $\begin{array}{c}\text { Injection Volume } \\
{[\mathrm{pl}]}\end{array}$ & $\begin{array}{c}\text { Susceptometer } \\
{[\%]}\end{array}$ & $\begin{array}{c}\text { Resonance Bridge } \\
{[\%]}\end{array}$ & $\begin{array}{c}\text { Inductance Sensor } \\
{[\%]}\end{array}$ \\
\hline 84.1 & 0 & 0 & 0 \\
56.1 & 0 & 0 & 0 \\
28.0 & 0 & 8 & 1 \\
14.0 & 11 & 35 & 20 \\
7.01 & 43 & 47 & 38 \\
\hline
\end{tabular}

\subsection{Sensitivity}

In Figure 5 the measured transmission sequence to determine the SNR values is shown for some exemplary injection volumes. As the injection volume and therefore the signal at the sensor decreases the relative noise grows. This expected behaviour can also be observed in the SNR values derived from measurements with injection volumes ranging from $7.01 \mu \mathrm{l}$ to $84.1 \mu \mathrm{l}$, found in Figure 6. 


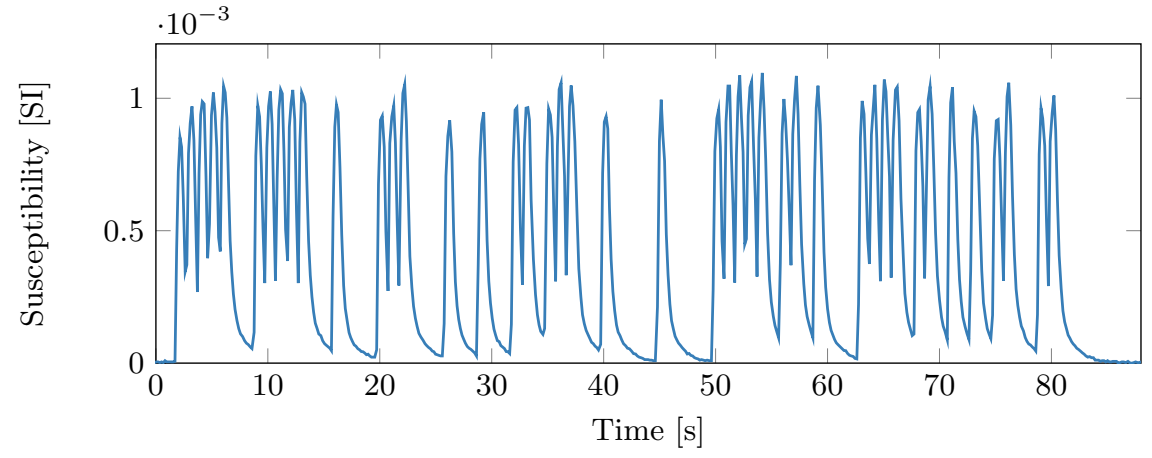

(a) Susceptometer

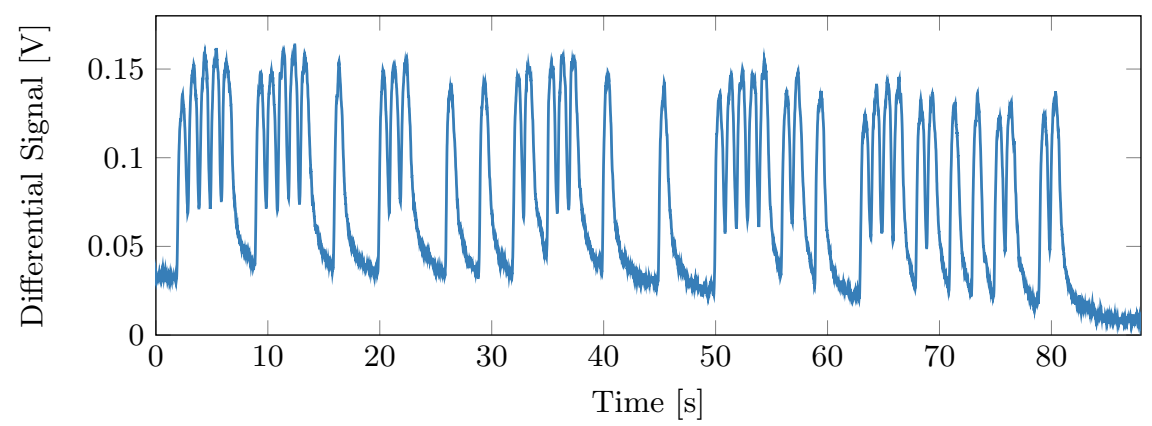

(b) Resonance Bridge

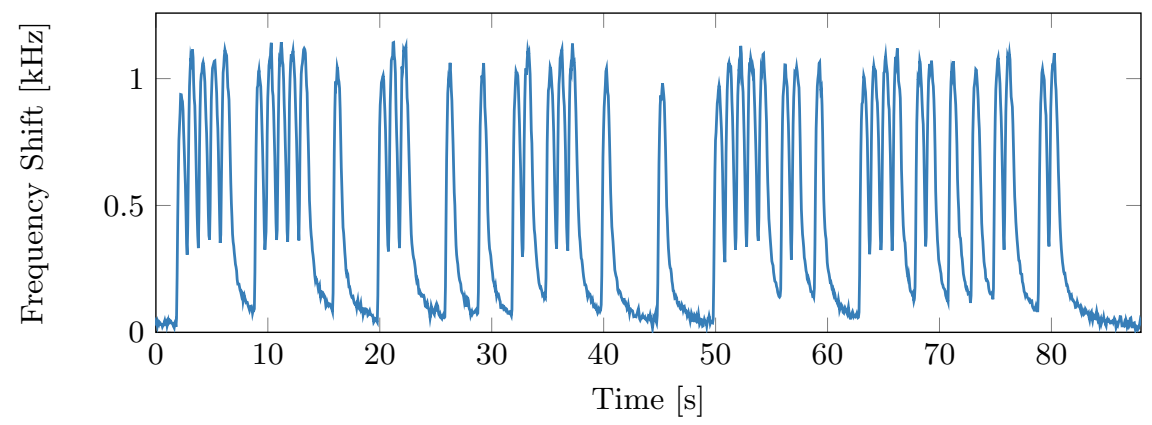

(c) Inductance Sensor

Fig. 4. Transmission of the 80-bit sequence described in (3). Logical high levels were coded as $84.1 \mathrm{\mu l}$ SPION injections. All three sensor devices were used in turn and the received signal filtered to achieve an equal sample rate of approx. $10 \mathrm{Sas}^{-1}$. 
The inductance sensor has a higher SNR value than the resonance bridge for all measurements. This is consistent with the results regarding BER in Table 1 and the high level of observed noise for the resonance bridge in Figure 5. The susceptometers SNR exceeds both of the other devices.

\subsection{Symbol Interval Variation}

BERs for each device were calculated for reduced symbol intervals at two different injection volumes $(14.0 \mu \mathrm{l}$ and $28.0 \mu \mathrm{l})$. With an injection volume of $28.0 \mu \mathrm{l}$ the advantage of higher sample rates for the resonance bridge and inductance sensor becomes dominant over the reduced sensitivity as the symbol interval decreases (see Figure 7a). This results in similar BER values for all three devices.

Figure $7 \mathrm{~b}$ shows the calculated BERs at an injection volume of $14.0 \mu \mathrm{l}$. At this lower concentration the resonance bridge consistently shows frequent bit errors due to the low sensitivity of the device. BERs for the inductance sensor however are again close to the susceptometers values.

In comparison to the higher injection volume of $28.0 \mu \mathrm{l}$ the BERs for both the inductance sensor and the susceptometer are improved at symbol intervals of less than $0.6 \mathrm{~s}$. This is due to reduced intersymbol interference (ISI) with lower injection volumes. For longer symbol intervals the influence of ISI is less relevant as more time is allowed for the SPION concentration in the channel to return to zero.

\section{Conclusion}

A new receiver principle using an inductance sensor to detect SPIONs was implemented. The proposed sensor is intended to replace the two previously used detector systems: The susceptometer with an insufficent sample rate in addition to a fixed measurement aperture and the resonance bridge, which relies on a manual tuning procedure. Random bit sequences were transmitted to successfully prove the sensor setup as a receiver.

Two devices (inductance sensor and resonance bridge) were tested in comparison to the reference device (susceptometer) with respect to sensitivity and bit-error rate with given testbed parameters.

The new devices both provide a sufficient sample rate. However, the inductance sensor has a higher SNR and therefore a better sensitivity than the previously constructed resonance bridge. In addition, the BER achieved with the inductance sensor are consistent with the improved SNR values and no manual tuning procedure as for the resonance bridge, with an impact on measurement reproducibility, is necessary. Furthermore, in scenarios with reduced symbol intervals the higher sample rate of the inductance sensor results in BERs similar to the susceptometers values, outweighing the influence of a lower SNR. In conclusion the inductance sensor, although not yet achieving the SNR values of the susceptometer, is an improvement in respect to setups with a high bit rate (requiring a high sample rate) or large transmission channels. 


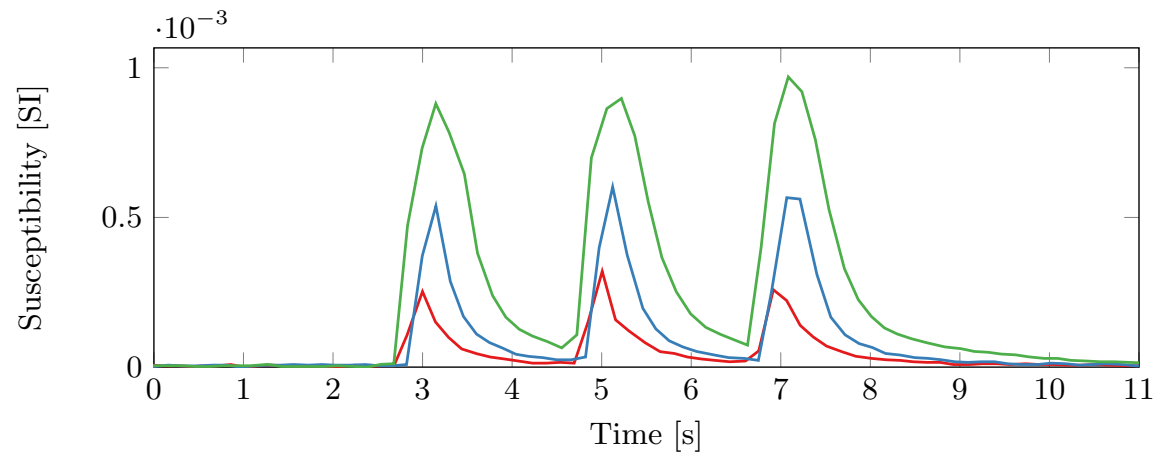

(a) Susceptometer

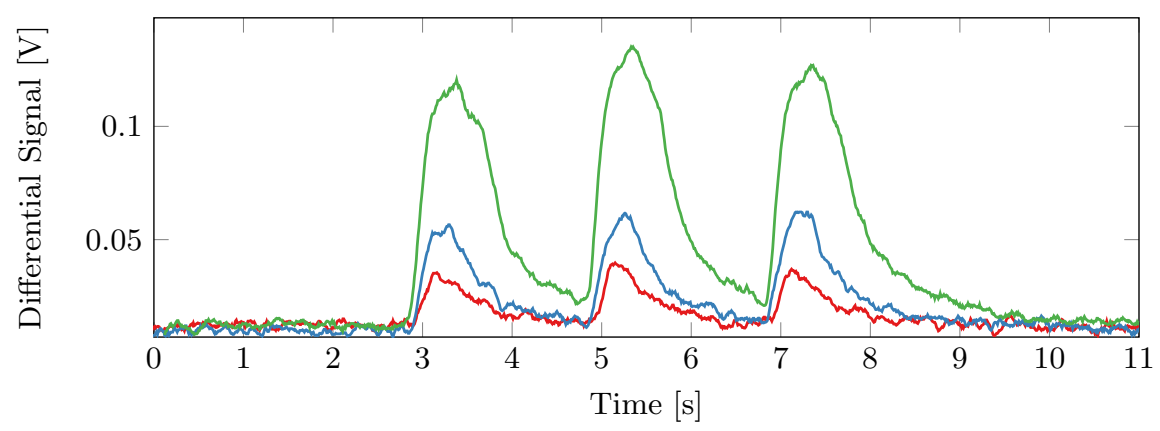

(b) Resonance Bridge

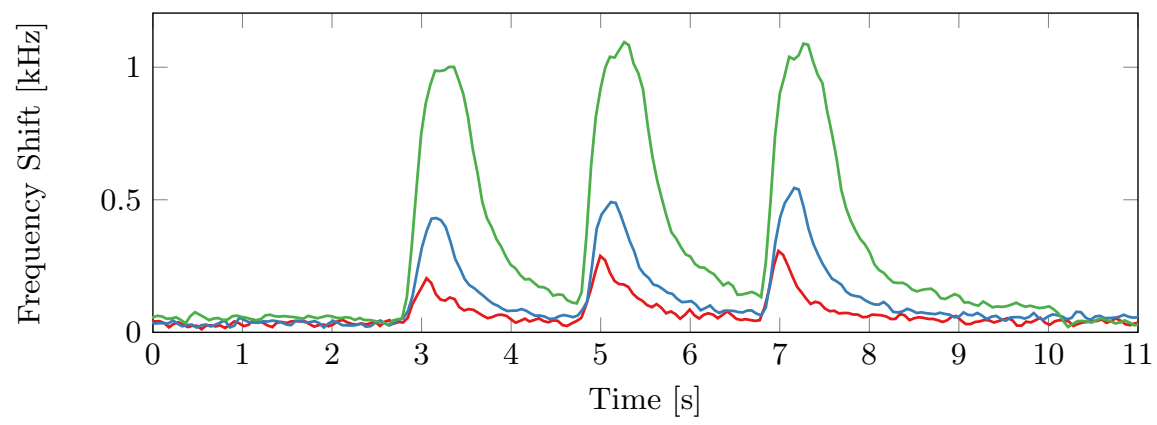

(c) Inductance Sensor

Fig. 5. Transmission of three individual pulses with varying injection volumes $(84.1 \mu \mathrm{l}$ in green, $28.0 \mu \mathrm{l}$ in blue, $14.0 \mathrm{\mu l}$ in red). The signal-to-noise ratio is derived by averaging the three local maxima in relation to the noise amplitude during no transmission. 


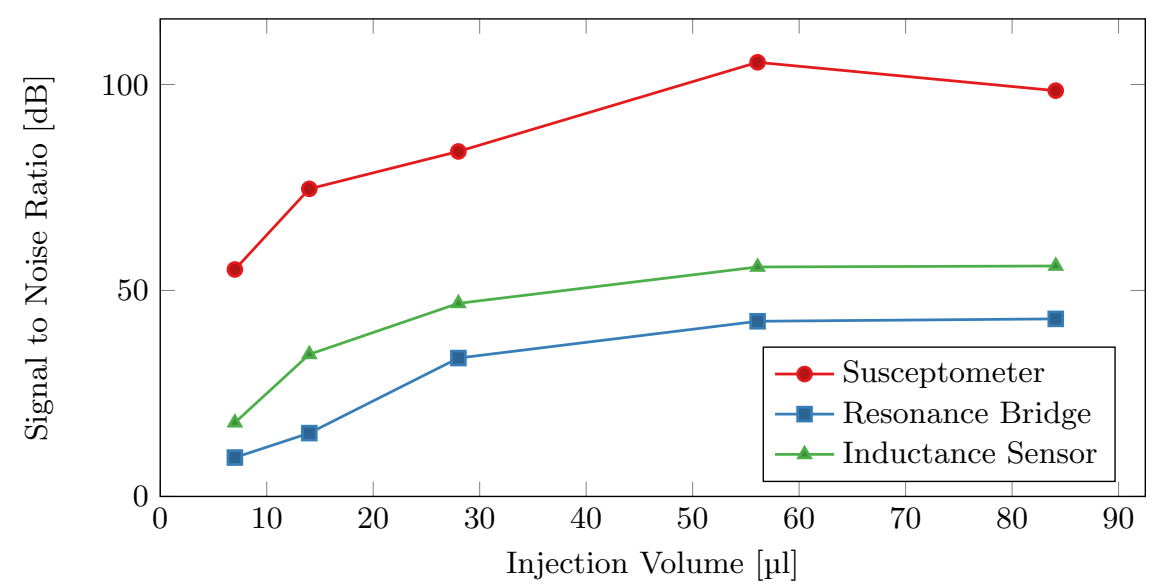

Fig. 6. SNR for different injection volumes derived from three individually transmitted pulses.

Various steps will be taken to further improve the future performance of the device: In respect of sensitivity and temporal resolution, results could be optimised by employing a different geometry for the detector coils, such as a reduced length to achieve a smaller measurement volume and thus increasing the spatial precision. Furthermore, noise might be reduced by providing the individual coils with an outer shielding or implementing a setup with multiple receivers on one channel used in parallel for averaging. Such a multi-channel receiver, in combination with a planar coil design placed tangentially to the transmission channel, would also provide the possibility of increased data rates with geometrical coding (left/right, angular) of SPION injection. Finally, in combination with a more in-depth analysis of physical channel phenomena, software on the receiver side with an elaborate decoding scheme will be developed.

\section{Acknowledgements}

This work was supported in part by the Emerging Fields Initiative (EFI) of the Friedrich-Alexander-Universität Erlangen-Nürnberg (FAU), the STAEDTLERStiftung, and the German Federal Ministry of Education and Research (BMBF), project MAMOKO.

\section{References}

1. Bartunik, M., Lübke, M., Unterweger, H., Alexiou, C., Meyer, S., Ahmed, D., Fischer, G., Wicke, W., Jamali, V., Schober, R., Kirchner, J.: Novel receiver for superparamagnetic iron oxide nanoparticles in a molecular communication setting. In: Proceedings of the Sixth Annual ACM International Conference on 


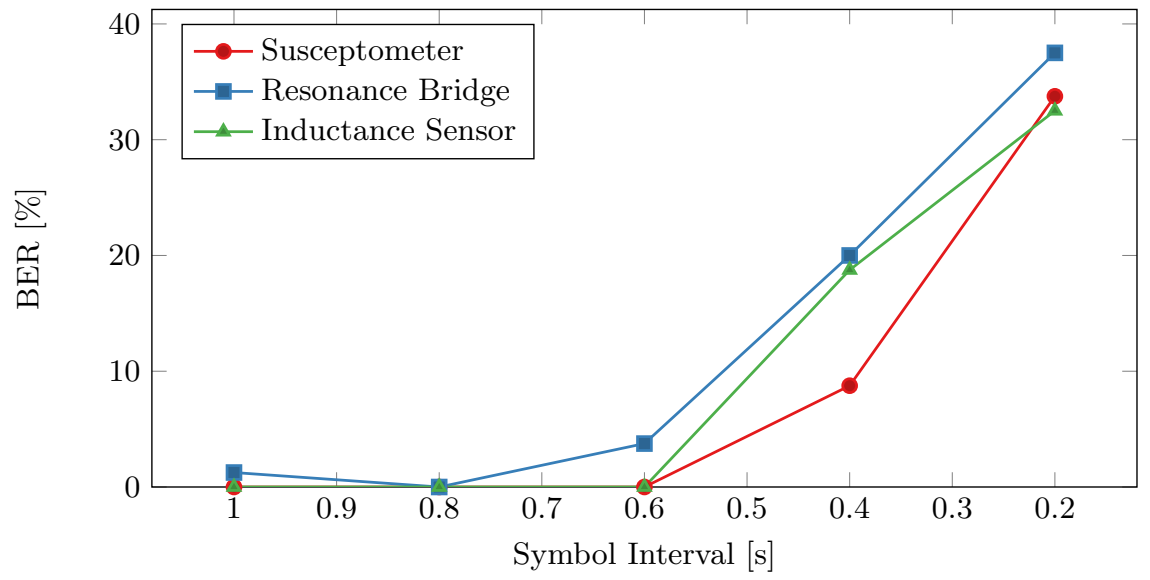

(a) Injection of $28.0 \mathrm{\mu l}$

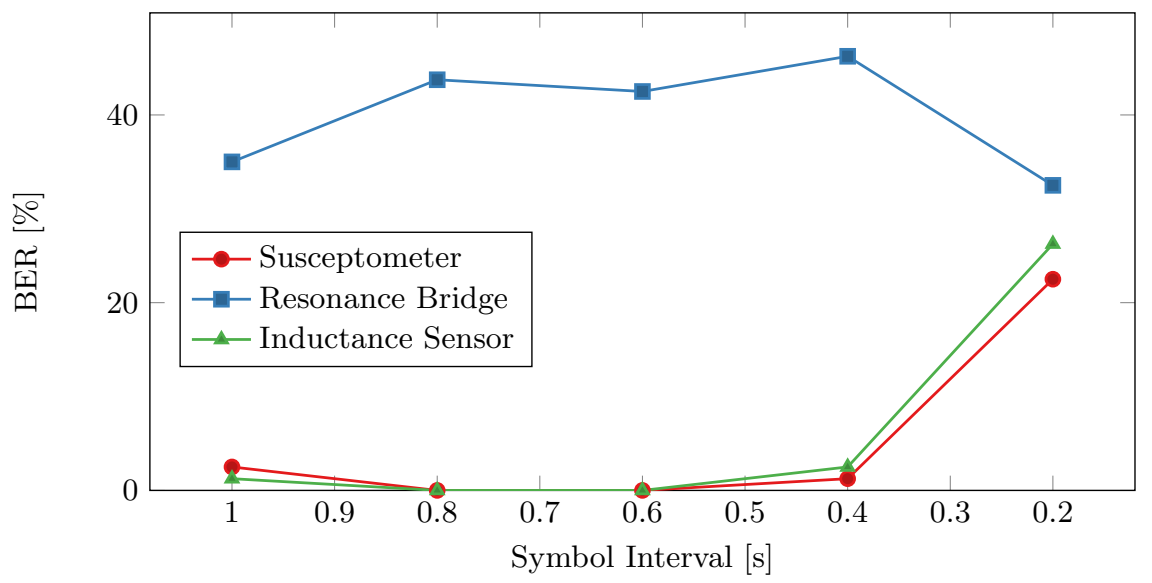

(b) Injection of $14.0 \mathrm{pl}$

Fig. 7. BERs for various symbol intervals. In Figure (a) $28.0 \mu \mathrm{l}$ of SPIONs were injected for each set bit of the transmission, respectively $14.0 \mathrm{\mu l}$ in Figure (b). 
Nanoscale Computing and Communication - NANOCOM '19. ACM Press (2019). https://doi.org/10.1145/3345312.3345483

2. Farsad, N., Guo, W., Eckford, A.W.: Tabletop molecular communication: Text messages through chemical signals. PLoS ONE 8(12), e82935 (Dec 2013). https://doi.org/10.1371/journal.pone.0082935

3. Farsad, N., Pan, D., Goldsmith, A.: A novel experimental platform for in-vessel multi-chemical molecular communications. In: GLOBECOM 2017 - 2017 IEEE Global Communications Conference. IEEE (Dec 2017). https://doi.org/10.1109/glocom.2017.8255058

4. Grebenstein, L., Kirchner, J., Peixoto, R.S., Zimmermann, W., Irnstorfer, F., Wicke, W., Ahmadzadeh, A., Jamali, V., Fischer, G., Weigel, R., Burkovski, A., Schober, R.: Biological optical-to-chemical signal conversion interface: A smallscale modulator for molecular communications. IEEE Transactions on NanoBioscience 18(1), 31-42 (Jan 2019). https://doi.org/10.1109/tnb.2018.2870910

5. Jamali, V., Ahmadzadeh, A., Wicke, W., Noel, A., Schober, R.: Channel modeling for diffusive molecular communication - a tutorial review. Proceedings of the IEEE 107(7), 1256-1301 (Jul 2019). https://doi.org/10.1109/jproc.2019.2919455

6. Koo, B.H., Lee, C., Yilmaz, H.B., Farsad, N., Eckford, A., Chae, C.B.: Molecular MIMO: From theory to prototype. IEEE Journal on Selected Areas in Communications 34(3), 600-614 (Mar 2016). https://doi.org/10.1109/jsac.2016.2525538

7. Krishnaswamy, B., Austin, C.M., Bardill, J.P., Russakow, D., Holst, G.L., Hammer, B.K., Forest, C.R., Sivakumar, R.: Time-elapse communication: Bacterial communication on a microfluidic chip. IEEE Transactions on Communications 61(12), 5139-5151 (Dec 2013). https://doi.org/10.1109/tcomm.2013.111013.130314

8. Matveev, A.N.: Electricity and Magnetism. Mir Publishers Moscow (1986)

9. Nakano, T., Eckford, A.W., Haraguchi, T.: Molecular Communication. Cambridge University Press (2009). https://doi.org/10.1017/cbo9781139149693

10. Unterweger, H., Kirchner, J., Wicke, W., Ahmadzadeh, A., Ahmed, D., Jamali, V., Alexiou, C., Fischer, G., Schober, R.: Experimental molecular communication testbed based on magnetic nanoparticles in duct flow. In: 2018 IEEE $19^{\text {th }}$ International Workshop on Signal Processing Advances in Wireless Communications (SPAWC). pp. 1-5 (Jun 2018). https://doi.org/10.1109/SPAWC.2018.8446011

11. Wang, L., Farsad, N., Guo, W., Magierowski, S., Eckford, A.W.: Molecular barcodes: Information transmission via persistent chemical tags. In: 2015 IEEE International Conference on Communications (ICC). IEEE (Jun 2015). https://doi.org/10.1109/icc.2015.7248469 